\title{
DIFFERENTIABLE PERIODIC MAPS
}

\author{
BY P. E. CONNER AND E. E. FLOYD ${ }^{1}$ \\ Communicated by Deane Montgomery, November 20, 1961
}

1. The bordism groups. This note presents an outline of the authors' efforts to apply Thom's cobordism theory [6] to the study of differentiable periodic maps. First, however, we shall outline our scheme for computing the oriented bordism groups of a space [1]. These preliminary remarks bear on a problem raised by Milnor [4]. A finite manifold is the finite disjoint union of compact connected manifolds with boundary each of which carries a $C^{\infty}$-differential structure. The boundary of a finite manifold, $B^{n}$, is denoted by $\partial B^{n}$. A closed manifold is a finite manifold with void boundary. We now define the oriented bordism groups of a pair $(X, A)$.

An oriented singular manifold in $(X, A)$ is a map $f:\left(B^{n}, \partial B^{n}\right)$ $\rightarrow(X, A)$ of an oriented finite manifold. Such a singular manifold bords in $(X, A)$ if and only if there is a finite oriented manifold $W^{n+1}$ and a map $F: W^{n+1} \rightarrow X$ such that $B^{n} C \partial W^{n+1}$ as a finite regular submanifold whose orientation is induced by that of $W^{n+1}$ and such that $F \mid B^{n}=f, F\left(\partial W^{n+1}-B^{n}\right) \subset A$. From two such oriented singular manifolds $\left(B_{1}^{n}, f_{1}\right)$ and $\left(B_{2}^{n}, f_{2}\right)$ a disjoint union $\left(B_{1}^{n} \cup B_{2}^{n}, f_{1} \cup f_{2}\right)$ is formed with $B_{1}^{n} \cap B_{2}^{n}=\varnothing$ and $f_{1} \cup f_{2} \mid B_{i}^{n}=f_{i}, i=1,2$. Obviously $-\left(B^{n}, f\right)$ $=\left(-B^{n}, f\right)$. We say that two singular manifold $\left(B_{1}^{n}, f_{1}\right)$ and $\left(B_{2}^{n}, f_{2}\right)$ are bordant in $(X, A)$ if and only if the disjoint union $\left(B_{1}^{n} \cup-B_{2}^{n}, f_{1} \cup f_{2}\right)$ bords in $(X, A)$. By the well-known angle straightening device [5] this is shown to form an equivalence relation. The oriented bordism class of $\left(B^{n}, f\right)$ is written $\left[B^{n}, f\right]$ and the collection of all such bordism classes is $\Omega_{n}(X, A)$. An abelian group structure is imposed on $\Omega_{n}(X, A)$ by disjoint union, and then following Atiyah we refer to $\Omega_{n}(X, A)$ as an oriented bordism group of $(X, A)$. The weak direct sum $\Omega_{*}(X, A)$ $=\sum_{0}^{\infty} \Omega_{n}(X, A)$ is a graded right module over the oriented Thom cobordism ring $\Omega$. For any $f:\left(B^{n}, \partial B^{n}\right) \rightarrow(X, A)$ and any closed oriented manifold $V^{m}$ the module product is given by $\left[B^{n}, f\right]\left[V^{m}\right]$ $=\left[B^{n} \times V^{m}, g\right]$ where $g(x, y)=f(x)$. For any map $\phi:(X, A) \rightarrow(Y, B)$ there is an induced homomorphism $\phi_{*}: \Omega_{n}(X, A) \rightarrow \Omega_{n}(Y, B)$ given by $\phi_{*}\left(\left[B^{n}, f\right]\right)=\left[B^{n}, \phi f\right]$. There is also $\partial_{*}: \Omega_{n}(X, A) \rightarrow \Omega_{n-1}(A)$ given by $\partial_{*}\left(\left[B^{n}, f\right]\right)=\left[\partial B^{n}, f \mid \partial B^{n} \rightarrow A\right]$. Actually $\phi_{*}: \Omega_{*}(X, A) \rightarrow \Omega_{*}(Y, B)$ and $\partial_{*}: \Omega_{*}(X, A) \rightarrow \Omega_{*}(A)$ are $\Omega$-module homomorphisms of degree 0 and -1 .

1 The authors are both Alfred P. Sloan Fellows. This work was partially supported by Air Research and Development Command Contract No. AF49(638)-72, Project No. 47500 . 
(1.1) Theorem. On the category of all pairs and maps the covariant functor $\left\{\Omega_{n}(X, A), \phi_{*}, \partial_{*}\right\}$ satisfies the first six axioms of EilenbergSteenrod for a homology theory. For a single point, however, $\Omega_{n}(p) \simeq \Omega_{n}$, the oriented Thom cobordism group.

We may define reduced groups ${ }^{\sim} \Omega_{n}(X)$ for $X \neq \varnothing$ by letting $\phi(X)=p$ and setting $\sim \Omega_{n}(X)=\operatorname{ker}\left(\Omega_{n}(X) \rightarrow \Omega_{n}(p)\right)$. By the usual argument $\Omega_{n}(X) \simeq \Omega_{n}+\sim \Omega_{n}(X)$. On the category of finite CW-pairs the strong excision theorem holds. Thus for a $k$-sphere we see by the axioms: $\Omega_{n}\left(S^{k}\right) \simeq \Omega_{n-k}$ and $\Omega_{n}\left(S^{k}\right)=\Omega_{n}+\Omega_{n-k}$.

There is a natural homomorphism $\mu: \Omega_{n}(X, A) \rightarrow H_{n}(X, A)$ obtained by assigning to every pair $\left(B^{n}, f\right)$ the image of the orientation class under $f_{*}: H_{n}\left(B^{n}, \partial B^{n} ; Z\right) \rightarrow H_{n}(X, A ; Z)$. For $(X, A)$ a finite $C W$-pair we may use the Cartan-Eilenberg construction of a spectral sequence to show [2].

(1.2) Theorem. For every finite $\mathrm{CW}$-pair $(X, A)$ there is a spectral sequence $\left\{E_{p, q}^{1}, d_{r}\right\}$ and a filtration $\Omega_{n}(X, A)=J_{n, 0} \supset J_{n-1,1} \supset \ldots$ $\supset J_{0, n} \supset 0$ with $E_{p, q}^{1} \simeq C_{p}\left(X, A ; \Omega_{q}\right), E_{p, q}^{2} \simeq H_{p}\left(X, A ; \Omega_{q}\right)$ and $E_{p, q}^{\infty}$ $\simeq J_{p, q} / J_{p-1, q+1}$.

Obviously $J_{p, q}=\operatorname{im}\left(\Omega_{p+q}\left(X^{p} \cup A, X^{p-1} \cup A\right) \rightarrow \Omega_{p+q}(X, A)\right)$. For $r \geqq 2$ the spectral sequence is independent of the CW-decomposition. The edge homomorphism $\Omega_{n}(X, A) \rightarrow J_{n, 0} / J_{n-1,1}=E_{n, 0}^{\infty} \subset E_{n, 0}^{2}$ $=H_{n}(X, A ; Z)$ naturally coincides with $\mu: \Omega_{n}(X, A) \rightarrow H_{n}(X, A ; Z)$. Furthermore there is a natural pairing

$$
E_{p, q}^{r} \otimes \Omega_{s} \rightarrow E_{p, q+s}^{r}
$$

for which $d_{r}$ may be regarded as an $\Omega$-module homomorphism. The pairing naturally agrees with the $\Omega$-module structure on $\Omega_{*}(X, A)$.

It is difficult to analyze the bordism functor directly, thus we follow Atiyah and turn to a problem in stable homotopy. We denote by $M S O$ the stable Thom object (or spectrum) consisting of the sequence $\cdots M S O(k), M S O(k+1) \cdots$ of Thom spaces together with the specific imbeddings of the suspension $S(M S O(k))$ in $M S O(k+1)$ given in [6]. For any integer $n$ we define $\Omega^{n}(X, A)$ (the cobordism group) to be the common value of $\left[S^{k}(X, A), M S O(k+n)\right]$ for $k$ large. Thus we obtain $\left\{\Omega^{n}(X, A), \phi^{*}, \delta^{*}\right\}$ a contravariant functor which on the category of finite $C W$-pairs satisfies the first six axioms of Eilenberg-Steenrod for cohomology. For a point $\Omega^{n}(p)$ $\simeq \Omega_{-n}$. There is the Thom-Atiyah duality theorem.

(1.3) THEOREM. If $X-A$ is an oriented k-manifold there is a canonical isomorphism $u: \Omega_{n}(X-A) \simeq \Omega^{k-n}(X, A)$. 
This theorem can be followed up along the lines suggested by Spanier-Whitehead duality. Eventually it is shown that

(1.4) Theorem. Let $X$ be a finite CW-complex with a weak $n$-dual, $D_{n}(X) \subset S^{n}$, with $n$ even. The bordism spectral sequence $E_{p, e}^{r}$ of $X$ and the cobordism spectral sequence $E_{r}^{p, q}$ for the pair $\left(S^{n}, D_{n}(X)\right)$ are dual. That is, there are isomorphisms $u: E_{p, q}^{r} \simeq E_{r}^{n-p-1,-q}$ which commute with the differentials and such that $u: E^{\infty} \rightarrow E_{\infty}$ is induced by $\Omega_{*}(X)$ $\simeq \Omega^{*}\left(S^{n}, D_{n}(X)\right)$.

From this follows directly the corollary.

(1.5) Corollary. Let $E_{p, q}^{r}$ be the bordism spectral sequence of a finite CW-pair, then for $r \geqq 2$ and all $(p, q)$ the image of $d_{r}: E_{p, q}^{r}$ $\rightarrow E_{p-r, q+r-1}^{r}$ is a finite group of odd order.

The corollary is first shown for a cobordism spectral sequence. In this case Wall's observation that modulo the class of torsion groups of odd order $M S O$ can be regarded as a product of Eilenberg-MacLane spaces gives the remark immediately [7]. From (1.5), the fact that $\Omega$ has no odd torsion, and the behavior of the $\Omega$-module structure in the bordism spectral sequence we see easily

(1.6) Theorem. If $(X, A)$ is a finite $\mathrm{CW}$-pair then the bordism spectral sequence collapses (is trivial) if and only if $\mu: \Omega_{n}(X, A)$ $\rightarrow H_{n}(X, A ; Z)$ is surjective for all $n \geqq 0$.

Obviously there is an isomorphism $\Omega_{n}(X, A) \simeq \sum_{p+q} H_{p}\left(X, A ; \Omega_{q}\right)$ modulo the class of torsion groups of odd order. If we combine (1.5) and (1.6) and the known structure of $\Omega$ we see

(1.7) Corollary. If $(X, A)$ is a finite $\mathrm{CW}$-pair for which $H_{*}(X, A ; Z)$ has no odd torsion then $\mu: \Omega_{n}(X, A) \rightarrow H_{n}(X, A ; Z)$ is surjective for $n \geqq 0$ and $\Omega_{n}(X, A) \simeq \sum_{p+q} H_{p}\left(X, A ; \Omega_{q}\right)$.

This last isomorphism is not natural. This, together with the following remark, sheds some light on a problem posed by Steenrod which asks if an integral homology class on a complex can be realized as the image of the orientation class of a closed manifold under a suitable map into the complex.

(1.8) Corollary. Let $(X, A)$ be a finite $\mathrm{CW}$-pair with $H_{2 k+1}(X, A ; Z)$ $=0$ for $k \geqq 0$, then $\mu: \Omega_{n}(X, A) \rightarrow H_{n}(X, A ; Z)$ is surjective for $n \geqq 0$.

We might also take ${ }^{\sim} H_{2 k}(X, A ; Z)=0$ for $k \geqq 0$ and the same follows. It can be shown that if $H_{*}(X, A ; Z)$ has no torsion then $\Omega_{*}(X, A)$ has an $\Omega$-base, thus it is a free $\Omega$-module. Clearly unoriented bordism 
groups $\mathfrak{T}_{n}(X, A)$ can also be defined by ignoring orientation. Every element has order 2 of course and $\mathfrak{T}_{*}(X, A)$ is a graded right $\mathfrak{T}$ module over the unoriented cobordism ring $\mathscr{N}$. In this case however:

(1.9) Theorem. For a finite $\mathrm{CW}$-pair $\mathfrak{N}_{*}(X, A)$ is a free graded

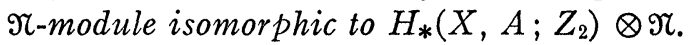

The bordism spectral sequence always collapses in the unoriented case since $\Re_{n}(X, A) \rightarrow H_{n}\left(X, A ; Z_{2}\right)$ is always surjective [6]. Obviously there is a reduction homomorphism $r: \Omega_{n}(X, A) \rightarrow \mathfrak{T}_{n}(X, A)$ and we can show a generalization of the Rochlin exact sequence:

(1.10) Theorem. For every finite CW-pair the sequence $\Omega_{n}(X, A)$ $\rightarrow^{2} \Omega_{n}(X, A) \rightarrow^{r} \mathfrak{T}_{n}(X, A)$ is exact.

Naturally the question will arise as to whether or not the bordism class of a map can be characterized by algebraic invariants, which would then be the analogues of Stiefel-Whitney numbers and Pontrjagin numbers. Let $w_{0}, w_{1}, \cdots, w_{n}$ be the Stiefel-Whitney classes of $M^{n}$. Let $\sigma \in H_{n}\left(M^{n} ; Z_{2}\right)$ be the fundamental class of the closed manifold $M^{n}$. Let $f: M^{n} \rightarrow X$ be a map and let $h_{k} \in H^{k}\left(X ; Z_{2}\right)$ be a cohomology class. For any $i_{1}+i_{2}+\cdots+i_{j}=n-k$ we obtain a Whitney number of the map $f$ by $\left\langle w_{i_{1}} \cdot w_{i_{2}} \cdots w_{i_{j}} \cdot f^{*}\left(h_{k}\right), \sigma\right\rangle \in Z_{2}$. The collection of all Whitney numbers of $f$ formed by taking all classes in $H^{*}\left(X ; Z_{2}\right)$ and all partitions uniquely determines the unoriented bordism class $\left[M^{n}, f\right]_{2} \in \mathscr{N}_{n}(X)$. By analogy if $M^{n}$ is oriented the Pontrjagin numbers of the map $f: M^{n} \rightarrow X$ are also defined.

(1.11) Theorem. If $X$ is a finite $\mathrm{CW}$-complex and if all torsion classes in $H_{*}(X ; Z)$ has order exactly 2 , then $\left[M^{n}, f\right] \in \Omega_{n}(X)$ is uniquely determined by giving all the Pontrjagin numbers and all the Whitney numbers of the map $f$.

We now turn to interpretations.

2. Bordism classification of bundles. Let $G$ denote a compact Lie group, possibly finite, with classifying space $B(G)$. Although $B(G)$ is not a finite CW-complex all the results of $\$ 1$ apply to it. Now what is $\Omega_{n}(B(G))$ ? An element is given by a map $f: M^{n} \rightarrow B(G)$ of a closed oriented $n$-manifold into $B(G)$. However if $f, g: M^{n} \rightarrow B(G)$ are homotopic, clearly $\left[M^{n}, f\right]=\left[M^{n}, g\right]$. Thus $\left[M^{n}, f\right]$ is really given by a preferred homotopy class of maps of $M^{n}$ into the classifying space. This, on the other hand, exactly determines a $G$-bundle over $M^{n}$. What we have actually is a cobordism classification of $G$-bundles over closed oriented manifolds. The fibre is immaterial. Now the 
reader may apply $\S 1$ to compute $\Omega_{*}(B(G))$ if $G=O(k), S O(k), U(k)$ or $\operatorname{Sp}(k)$. A similar meaning is given to $\mathfrak{T}_{*}(B(G))$.

Another interpretation is very useful when $G$ is finite. We shall restrict ourselves to cyclic transformations of prime order. Let $\left(T, M^{n}\right)$ be an orientation preserving, fixed point free diffeomorphism of prime period $p$ on a closed oriented manifold. We say that $\left(T, M^{n}\right)$ equivariantly bords if and only if there is a fixed point free orientation preserving diffeomorphism of period $p$ on a finite manifold $\left(\tau, B^{n+1}\right)$ for which $\left(\tau, \partial B^{n+1}\right)$ is equivariantly diffeomorphic to $\left(T, M^{n}\right)$ by an orientation preserving diffeomorphism. Note that no fixed points are allowed. Now from $\left(T_{1}, M_{1}^{n}\right)$ and $\left(T_{2}, M_{2}^{n}\right)$ a disjoint union $\left(T_{1} \cup T_{2}, M_{1}^{n} \cup M_{2}^{n}\right)$ is formed with $M_{1}^{n} \cap M_{2}^{n}=\varnothing$ and $T_{1} \cup T_{2} \mid M_{i}^{n}$ $=T_{i}, i=1,2$. Let $-\left(T, M^{n}\right)=\left(T,-M^{n}\right)$. Then $\left(T_{1}, M_{1}^{n}\right)$ is equivariantly bordant to $\left(T_{2}, M_{2}^{n}\right)$ if and only if $\left(T_{1} \cup T_{2}, M_{1}^{n} \cup-M_{2}^{n}\right)$ equivariantly bords. Let $\left[T, M^{n}\right]$ denote the bordism class and introduce an abelian group structure by disjoint union. Denote the result by $\Omega_{n}\left(B\left(Z_{p}\right)\right)$. This is valid notation since a free diffeomorphism of period $p,\left(T, M^{n}\right)$, determines a principal $Z_{p}$-bundle $M^{n} \rightarrow M^{n} / T$ which in turn must define an element in $\Omega_{n}\left(B\left(Z_{p}\right)\right)$. A map $f: V^{n}$

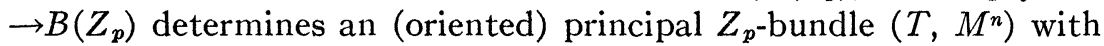
$M^{n} / T=V^{n}$. We can also consider $\mathfrak{N}_{n}\left(B\left(Z_{p}\right)\right)$ with the same interpretation. Now the $\Omega$-module structure is given by $\left[T, M^{n}\right]\left[V^{m}\right]$ $=\left[T^{\prime}, M^{n} \times V^{m}\right]$ where $T^{\prime}(x, y)=(T(x), y)$. Note for $p$ odd that $H_{*}\left(B\left(Z_{p}\right) ; Z_{2}\right) \simeq Z_{2}$ thus $\mathscr{T}_{*}\left(B\left(Z_{p}\right)\right) \simeq \mathscr{T}$. However $\mathfrak{T}_{*}\left(B\left(Z_{2}\right)\right)$ is a free graded $\mathscr{T}$-module with homogeneous $\mathscr{N}$-base given by the antipodal involutions of spheres $\left\{\left[A, S^{n}\right]_{2}\right\}, n \geqq 0$. In addition $\Omega_{*}\left(B\left(Z_{2}\right)\right)$ can be completely determined by (1.7). For $p$ odd, however, the situation is different. We shall give $\widetilde{\Omega}_{*}\left(B\left(Z_{p}\right)\right)$, which is exactly the $p$-torsion in $\Omega_{*}\left(B\left(Z_{p}\right)\right)$. Let $\lambda=\exp (2 \pi i / p)$. Let

$$
S^{2 k+1}=\left\{\left(z_{1}, z_{2}, \cdots, z_{k+1}\right) \mid \sum z_{i} \bar{z}_{i}=1\right\}
$$

and define $\left(T, S^{2 k+1}\right)$ by $T\left(z_{1}, \cdots, z_{k+1}\right)=\left(\lambda z_{1}, \cdots, \lambda z_{k+1}\right)$. The elements $\left[T, S^{2 k+1}\right]$ generate $\sim \Omega_{*}\left(B\left(Z_{p}\right)\right)$ as an $\Omega$-module. Milnor has shown [3] that $\Omega \bmod$ torsion is a polynomial ring with generators $\left[X^{4 r}\right] \in \Omega, 0 \leqq r<\infty$.

(2.1) Theorem. For an odd prime, $\sim \Omega_{*}\left(B\left(Z_{p}\right)\right)$ is the direct sum of cyclic groups. $C_{2 k+1, r_{1}}, \cdots, r_{s}$ with generators $\left[T, S^{2 k+1}\right]\left[X^{4 r_{1}}\right] \cdots\left[X^{4 r_{s}}\right]$, one for each $2 k+1$ and each $r_{1}, \cdots, r_{s}$ with $4 r_{i} \neq 2 p-2$ all $r_{i}$. The order of $C_{2 k+1, r_{1}}, \cdots, r_{s}$ is $p^{a+1}$ where $a(2 p-2)<2 k+1<(a+1)(2 p-2)$.

It is somewhat surprising that the order of the $p$-torsion becomes so large. While the bordism sequence of $B\left(Z_{p}\right)$ will collapse for any 
prime we must, for odd primes, turn to more geometric methods to obtain the structure theorem (2.1). The argument is based on the analysis of certain known maps of period $p$ on $C P(p-1)$, complex projective $(p-1)$-space.

3. Fixed point sets of involutions. In this section we shall quickly sketch some applications of the foregoing to the fixed point set of differentiable involutions on closed unoriented manifolds. Let

$$
L_{n}=\sum \Re_{m}(B(O(n-m)))
$$

for $n \geqq 0$. Now $\mathfrak{T}_{m}(B(O(n-m)))$ is the unoriented bordism group of $(n-m)$-plane bundles with structural group $O(n-m)$ over closed $m$-manifolds. We shall define a homomorphism $j_{*}: L_{n} \rightarrow \mathfrak{N}_{n-1}\left(B\left(Z_{2}\right)\right)$. We choose in each bordism class of $\Re_{m}(B(O(n-m)))$ a differentiable bundle and let $B \rightarrow M^{m}$ denote the associated $(n-m-1)$-sphere bundle on $M^{m}$. Since the antipodal map lies in the center of $O(n-m)$ there is a fixed point free fibre preserving involution $(T, B)$ which on each fibre reduces to the antipodal involution. Now we assign $[T, B]_{2}$ $\in \mathscr{N}_{n-1}\left(B\left(Z_{2}\right)\right)$ to the bordism class of the original vector bundle. This defines $j_{*}: \mathfrak{I}_{m}(B(O(n-m))) \rightarrow \mathfrak{Y}_{n-1}\left(B\left(Z_{2}\right)\right)$ and $j_{*}$ is extended linearly to $L_{n}$. We let $\mathfrak{N}_{n}=\mathfrak{N}_{n}(B(O(0)))$ and $j_{*}\left(\Re_{n}\right)=0$. Let $\left(T, M^{n}\right)$ be a differentiable involution on a closed manifold. Let $F^{m}, 0 \leqq m \leqq n$, be the union of the $m$-dimensional components of the fixed point set of $T$. Each $F^{m}$ is a closed regular submanifold of $M^{n}$. Let $N \rightarrow F^{m}$ be the normal $(n-m)$-bundle to $F^{m}$ in $M^{n}$ and let $\left[N \rightarrow F^{m}\right]_{2}$ $\in \Re_{n}(B(O(n-m)))$ be the bordism class of this bundle.

(3.1) TheOREM. For any differentiable involution on a closed $n$ manifold, $\left(T, M^{n}\right)$

$$
\sum_{0}^{n} j_{*}\left(\left[N \rightarrow F^{m}\right]_{2}\right)=0 \in \mathfrak{N}_{n-1}\left(B\left(Z_{2}\right)\right) .
$$

This is seen by introducing a Riemannian metric on $M^{n}$ in which $T$ is an isometry. Each $F^{m}$ is surrounded by a small invariant tube of normal geodesics whose boundary is the normal $(n-m-1)$-sphere bundle to $F^{m}$. Let $W^{n} C M^{n}$ be the finite manifold obtained by removing the interiors of all these normal tubes. Obviously $W^{n}$ is $T$-invariant, and $\left(T, \partial W^{n}\right)$ is the disjoint union of the involutions on the normal sphere bundles to the $F^{m}$. Obviously $\left(T, W^{n}\right)$ has no fixed points. As a corollary we shall show that the normal bundle to the fixed point set determines $\left[M^{n}\right]_{2} \in \mathfrak{N}_{n}$. For each $F^{m}$ let $B_{m}^{\prime} \rightarrow F^{m}$ be the Whitney join of a trivial 0 -sphere bundle with the normal sphere bundle of $F^{m}$. Thus $B^{\prime} \rightarrow F^{m}$ is an $(n-m)$-sphere bundle. Let $\left(T^{\prime}, B_{m}^{\prime}\right)$ 
be the bundle involution of this new bundle. Note that $B_{m}^{\prime} / T^{\prime}$ is a closed $n$-manifold.

(3.2) ThEOREM. If $\left(T, M^{n}\right)$ is an involution on a closed n-manifold then

$$
\left[M^{n}\right]_{2}=\sum_{0}^{n}\left[B_{m}^{\prime} / T^{\prime}\right]_{2}
$$

We let $B_{n}^{\prime} / T^{\prime}=F^{n}$. We define two involutions on $I \times M^{n}$ by $\tau_{1}(t, x)$ $=(1-t, x)$ and $\tau_{2}(t, x)=(1-t, T(x))$. We adjoin $I \times M^{n}$ to a copy of itself along the boundary by the equivariant diffeomorphism $m(1, x)$ $=(1, T(x)), m(0, x)=(0, x)$. We obtain an involution $\left(T^{\prime}, V^{n+1}\right)$ on a closed manifold. Now (3.2) follows by applying (3.1) to $\left(T^{\prime}, V^{n+1}\right)$. We note that (3.1) and (3.2) are entirely geometric in nature. We note for example that if we consider $(T, C P(n))$ given by $T\left[z_{1}, \cdots, z_{n+1}\right]=\left[\bar{z}_{1}, \cdots, \bar{z}_{n+1}\right]$ then the fixed point set is real projective space $P^{n}$. The tangent bundle to $P^{n}$ is equivalent to the normal bundle of $P^{n}$ in $C P(n)$. According to (3.2) we may use the tangent bundle of $P^{n}$ to determine $[C P(n)]_{2}$. Consider $P^{n} \times P^{n}$ and $(x, y) \rightarrow(y, x)$ with fixed point set $P^{n}=\Delta \subset P^{n} \times P^{n}$. Again the normal bundle to $\Delta$ is equivalent to the tangent bundle to $P^{n}$, thus if we use (3.2) once more we see $[C P(n)]_{2}=\left[P^{n} \times P^{n}\right]_{2}$. This is the geometric proof of a well-known fact [7].

(3.3) THEOREM. Let $\left(T, M^{n}\right)$ be an involution on a closed manifold. If for each $m$ all the Whitney classes of the normal bundle to $F^{m}$ vanish, then $\left[F^{m}\right]_{2}=0,0 \leqq m<n$ and $\left[M^{n}\right]_{2}=\left[F^{n}\right]_{2}$.

Since all the Whitney classes of the normal bundle to $F^{m}$ vanish $N \rightarrow F^{m}$ is bordant to the product bundle $F^{m} \times R^{n-m} \rightarrow F^{m}$, thus by (3.1) $\sum_{0}^{n-1}\left[A, S^{n-m-1}\right]_{2}\left[F^{m}\right]_{2}=0$. However $\left[A, S^{k}\right]_{2}$ forms an $\Re$-base of $\mathscr{Y}_{*}\left(B\left(Z_{2}\right)\right)$ thus $\left[F^{n}\right]_{2}=0,0 \leqq m<n$. The rest is a simple consequence of (3.2). Dually we have

(3.4) THEOREM. Let $\left(T, M^{n}\right)$ be a differentiable involution with a connected fixed point. If all the Stiefel-Whitney classes of the tangent bundle of the fixed point set vanish, then $\left[M^{n}\right]_{2}=0$.

A more specific type of result is

(3.5) Theorem. Let $\left(T, M^{n}\right)$ be an involution whose fixed point set is the disjoint union of a point and a $k$-sphere, then $k=0,1,2,4$, or 8 , $n=2 k$ and $M^{2 k}$ is $\bmod Z$ cobordant to the appropriate projective plane.

Suppose all the Whitney classes of the normal bundle to $S^{k}$ vanish, 
then by (3.3) it follows that single point cobords mod 2. Thus we see that the $k$ th Whitney class of $N \rightarrow S^{k}$ is nonzero, so $k=0,1,2,4$ or 8 . Next (3.2) must be used heavily to finish the proof. We cannot indicate here the proof of

(3.6) THEOREM. For each integer $k \geqq 0$ there is an integer $\phi(k)$ such that if $\left(T, M^{n}\right)$ is an involutiion on a noncobording manifold with $n>\phi(k)$ then some component of the fixed point set has dimension greater than $k$.

We are not able to estimate $\phi(k)$ in general; however there is a more specific result of this type.

(3.7) Theorem. Let $\left(T, M^{2 n}\right)$ be an involution on a closed manifold with $\chi\left(M^{2 n}\right)=1 \bmod 2$, then there is a component of the fixed point set which has dimension at least $n$.

We now turn to $\left(Z_{2}\right)^{k}$, the $k$-fold direct sum of $Z_{2}$ with itself. A stationary point of a group acting is a point left fixed by the entire group.

(3.8) Theorem. Let $\left(\left(Z_{2}\right)^{k}, M^{n}\right)$ be a differentiable action on a closed manifold without stationary points, then $\left[M^{n}\right]_{2}=0$.

The proof uses (3.2) and induction over $k$. It would be extremely important to find any generalizations of

(3.9) Theorem. Let $\left(Z_{2}+Z_{2}, M^{n}\right)$ be a differentiable action with a finite number of stationary points, then the class $\left[M^{n}\right]_{2}$ will belong to the subring of $\mathfrak{x}$ generated by $\left[P^{2}\right]_{2}$, the cobordism class of the real projective plane.

In closing we might note that by considering all differentiable involutions of closed manifolds $\left(T, M^{n}\right)$ we define an unrestricted bordism group of involutions $I_{n}\left(Z_{2}\right)$. There is an $i_{*}: I_{n}\left(Z_{2}\right) \rightarrow L_{n}$ assigning to each $\left(T, M^{n}\right)$ the bordism classes $\sum_{0}^{n}\left[N \rightarrow F^{m}\right]_{2}$ of the normal bundles to the $F^{m}$. The sequence $0 \rightarrow I_{n}\left(Z_{2}\right) \rightarrow L_{n} \rightarrow \mathscr{T}_{n-1}\left(B\left(Z_{2}\right)\right) \rightarrow 0$ is split exact. Thus the unrestricted bordism group of involutions is computable.

4. Maps of odd prime period. An oriented action $\left(Z_{p}, M^{n}\right)$ is a differentiable orientation preserving action of $Z_{p}$ on an oriented manifold. We shall only consider $p$ an odd prime in this section. First we shall take up the analogue to (3.1). Let $S^{1}$ be the circle and let $\left(T^{\prime \prime}, S^{1}\right)$ be $T^{\prime \prime}(z)=\lambda z$ where $\lambda=\exp (2 \pi i / p)$. Let $\left(T, M^{n}\right)$ be an oriented action of $Z_{p}$ on $M^{n}$. Let $\left(T^{\prime \prime} \times T, S^{1} \times M^{n}\right)$ be the diagonal action $T^{\prime \prime} \times T(z, x)$ 
$=(\lambda z, T(x))$. This is always a free action of $Z_{p}$ defining an element of $\Omega_{n+1}\left(B\left(Z_{p}\right)\right)$.

(4.1). For any oriented action of $Z_{p}$ on $M^{n}$

$$
\left[T^{\prime \prime} \times T, S^{1} \times M^{n}\right]=\left[T^{\prime \prime}, S^{1}\right]\left[M^{n}\right]
$$

in $\Omega_{n+1}\left(B\left(Z_{p}\right)\right)$.

Consider now an oriented action $\left(Z_{p}, M^{n}\right)$ with $p$ an odd prime. Let $F^{m} \subset M^{n}$ be the union of the $m$-dimensional components of the fixed point set. Now $F^{m}=\varnothing$ if $n-m$ is odd. In general there is a canonical complex structure on the normal bundle to $F^{m}$ and $Z_{p}$ acts as a complex linear fibre preserving group of bundle maps on the normal bundle. Let $N_{c} \rightarrow F^{m}$ be the complex normal bundle and let $N_{c}+C \rightarrow F^{m}$ be the Whitney sum with a trivial complex line bundle. Let $B_{m}^{\prime} \rightarrow F^{m}$ be the resulting sphere bundle, and let $\left(T^{\prime}, B_{m}^{\prime}\right)$ be the resulting fibre preserving fixed point free action of $Z_{p}$ induced on this bundle space.

(4.2) TheOREM. Let $\left(Z_{p}, M^{n}\right)$ be an oriented action, then

$$
\sum_{m}\left[T^{\prime}, B_{m}^{\prime}\right]=\left[T^{\prime \prime}, S^{1}\right]\left[M^{n}\right]
$$

in $\Omega_{n+1}\left(B\left(Z_{p}\right)\right)$.

This is analogous to (3.2) and, with the aid of (4.1), can be seen from an analogous argument. A cursory examination of the bordism spectral sequence of $B\left(Z_{p}\right)$ shows

(4.3) Lemma. If $M^{n}$ is a closed oriented manifold, then $\left[T^{\prime \prime}, S^{1}\right]\left[M^{n}\right]$ $=0 \in \Omega_{n+1}\left(B\left(Z_{p}\right)\right)$ if and only if $\left[M^{n}\right] \in p \Omega_{n}$.

The determination of $\Omega_{*}\left(B\left(Z_{p}\right)\right)$ is based on these three results. For example we may define an oriented action $\left(Z_{p}, C P(p-1)\right)$ by $T\left(\left[z_{1}, z_{2}, \cdots, z_{p}\right]\right)=\left[z_{1}, \lambda z_{2}, \lambda^{2} z_{2}, \cdots, \lambda^{p-1} z_{p}\right]$. Now $\left(Z_{p}, C P(p-1)\right)$ has exactly $p$ fixed points. If we apply (4.2) and then (4.3) to $\left(Z_{p}, C P(p-1)\right)$ we can see that $\Omega_{2 p-1}\left(B\left(Z_{p}\right)\right)$ contains an element of order $p^{2}$.

We need in addition the idea of the local type of an isolated fixed point of $\left(Z_{p}, M^{n}\right)$. At a fixed point we can locally linearize the action which gives us an element of order $p$ in $S O(n)$. Two isolated fixed points $x_{0}$ and $x_{1}$ have the same type if and only if $T_{0}$ and $T_{1}$ are conjugate in $S O(n)$. All the fixed points in the above example have the same type.

(4.4) Theorem. Let $\left(Z_{p}, M^{n}\right)$ be an oriented action with a finite number of fixed points all of the same type, then the number of fixed 
points is a multiple of $p^{a+1}, a(2 p-2)<n-1<(a+1)(2 p-2)$ and $\left[M^{n}\right] \in p \Omega_{n}$ or $\left[M^{n}\right]=b[C P(p-1)]^{a} \bmod p \Omega_{n}$ for suitable $a$ and $b$.

The prime 3 has a special role since there are only two types of isolated fixed points and one is the negative of the other.

(4.5) Theorem. Let $\left(Z_{3}, M^{n}\right)$ be an oriented action with a finite number of fixed points, then $\left[M^{n}\right]$ represents in $\Omega / 3 \Omega$ an element of the subalgebra generated by $[C P(2)]$. If $\left[M^{n}\right] \neq 0$ in $\Omega / 3 \Omega$ there are at least $3^{a+1}$ fixed points with $4 a<n-1<4(a+1)$.

In general we do not precisely know how to specify the cobordism class of a manifold which admits an oriented action of $Z_{p}$ with a finite number of fixed points that need not be of the same type. We only have a partial result.

(4.6) Theorem. Let $\left(Z_{p}, M^{n}\right)$ be an oriented action with a finite number of fixed points, then every Pontrjagin number $s_{i_{1}, \ldots, i_{r}}\left[M^{n}\right]$ $=0 \bmod p$ whenever some $4 i_{s} \geqq 2 p-2$.

We now turn to elementary abelian $p$-groups $\left(Z_{p}\right)^{k}$, the $k$-fold direct sum of $Z_{p}$ with itself. There are three ideals in $\Omega$ which are important in connection with oriented actions of these groups. The first, $I(p) \subset \Omega$ is the ideal of cobordism classes in $\Omega$ all of whose Pontrjagin numbers are divisible by $p$. We shall settle the structure of $I(p)$ immediately. We say $\left[X^{4 k}\right]$ is a Milnor base element if and only if $s_{k}\left[X^{4 k}\right]=1$ if $2 k+1$ is not a prime power, or $s_{k}\left[X^{4 k}\right]=q$ if $2 k+1$ is a prime power $q^{r},[3]$.

(4.7) THEOREM. For each odd prime $p$ there are Milnor base elements $\left[Y^{2 p^{k}-2}\right], k=1,2, \cdots$ all of whose Pontrjagin numbers are divisible by $p$. Furthermore $I(p)$ is the ideal in $\Omega$ generated by $p \Omega$ together with the special Milnor base elements $\left\{\left[Y^{2 p^{k}-2}\right\}\right.$.

Thus the ideal $I(p)$ is rather large. For each $k \geqq 1$ let $I(p, k) \subset \Omega$ be the ideal of cobordism classes which contain a representative on which $\left(Z_{p}\right)^{k}$ acts without stationary points. We do not require effective action, thus $I(p, k) \subset I(p, k+1)$. We can see that $I(p, 1)=p \Omega$. We would very much like to know the structure of $I(p, k)$. An intermediate ideal is described as follows. Consider $\left(\left(Z_{p}\right)^{k}, T^{k}\right)$ the free action of $\left(Z_{p}\right)^{k}$ on the $k$-torus given by taking the $k$-fold product of $\left(T^{\prime \prime}, S^{1}\right)$ with itself. Now $\left[\left(Z_{p}\right)^{k}, T^{k}\right] \in \Omega_{k}\left(B\left(\left(Z_{p}\right)^{k}\right)\right)$. Recall that $\Omega_{*}\left(B\left(\left(Z_{p}\right)^{k}\right)\right)$ is a graded right $\Omega$-module, thus an ideal $J(p, k) \subset \Omega$ is defined to be the set of annihilators of the element $\left[\left(Z_{p}\right)^{k}, T^{k}\right]$. It is easy to see that $J(p, k) \subset I(p)$. It is less obvious that 
(4.8) Theorem. For any odd prime $p$ and $k \geqq 1$

$$
I(p, k) \subset J(p, k) \subset I(p) .
$$

For all we know it may happen that $I(p, k)=J(p, k)$. In any case more details about the structure of $J(p, k)$ would be interesting. The reader should explicitly note that if $\left(\left(Z_{p}\right)^{k}, M^{n}\right)$ is an oriented action without stationary points then all the Pontrjagin numbers of $M^{n}$ are divisible by $p$. This includes the case $p=2$. We close with two applications. The first is a Hopf-Samelson type result.

(4.9) Corollary. Let $G$ be a compact connected Lie group and $H a$ closed subgroup. If $[G / H]_{2} \neq 0$, then $H$ has maximal 2-rank. If $G / H$ is oriented and if for some prime $p$ there is a Pontrjagin number of $G / H$ not divisible by $p$, then $H$ has maximal p-rank. If $2[G / H] \neq 0$, then $H$ has maximal rank.

And we shall also include a corollary for algebraic geometry.

(4.10) COROLlaRy. If $V^{n}$ is a real nonsingular projective subvariety with real fold $F^{n} \subset V^{n}$, then $\left[V^{n}\right]_{2}=\left[F^{n} \times F^{n}\right]_{2}$.

This last is really a corollary of (3.2). We hope that we have succeeded in demonstrating that there are methods for studying differentiable periodic maps which really make effective use of the differentiability hypothesis. A detailed study of differentiable periodic maps will appear elsewhere.

\section{BIBLIOGRAPHY}

1. M. F. Atiyah, Bordism and cobordism, Proc. Cambridge Philos. Soc. 57 (1961), 200-208.

2. H. Cartan et al., Cartan seminar notes, Paris, 1950-1951.

3. J. W. Milnor, On the cobordism ring $\Omega^{*}$, Notices Amer. Math. Soc. 5 (1958), 457.

4. - A survey of cobordism, mimeographed notes, Princeton, 1961.

5. - Differentiable structures on homotopy spheres, mimeographed notes, Princeton, 1959.

6. R. Thom, Quelques propriêtés globales des variêtes différentiables, Comment. Math. Helv. 28 (1954), 17-86.

7. C. T. C. Wall, Determination of the cobordism ring, Ann. of Math. 72 (1960), 292-311.

The University of Virginia and

The Institute for Advanced Study 